\title{
LOW COST MULTI-SENSOR ROBOT LASER SCANNING SYSTEM AND ITS ACCURACY INVESTIGATIONS FOR INDOOR MAPPING APPLICATION
}

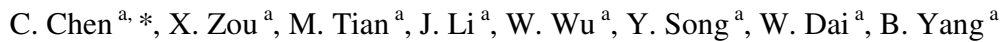 \\ ${ }^{\text {a }}$ State Key Laboratory of Information Engineering in Survey, Mapping and Remote Sensing, \\ Wuhan University, No. 129, Luoyu Road, Wuhan, PR China - chichen@whu.edu.cn
}

Commission II

KEY WORDS: Indoor Mapping, Laser Scanning, Multi-sensor, Robot

\begin{abstract}
:
In order to solve the automation of 3D indoor mapping task, a low cost multi-sensor robot laser scanning system is proposed in this paper. The multiple-sensor robot laser scanning system includes a panorama camera, a laser scanner, and an inertial measurement unit and etc., which are calibrated and synchronized together to achieve simultaneously collection of 3D indoor data. Experiments are undertaken in a typical indoor scene and the data generated by the proposed system are compared with ground truth data collected by a TLS scanner showing an accuracy of $99.2 \%$ below 0.25 meter, which explains the applicability and precision of the system in indoor mapping applications.
\end{abstract}

\section{INTRODUCTION}

In recent years, with the continuous improvement and maturity of the electronic, mechanical, and artificial intelligence science and technology, "Unmanned" has become a hot research and application topic. Unmanned is the trend of industrial manufacturing as well as our daily life. Autonomous driving (Thornton et al., 2017), and flying (Saripalli et al., 2015) has shown its great practical and research value. Driven by miniaturization, lightweight and intelligent of the remote sensing platform and sensor, fully automated data acquisition method is of great significance to the traditional photogrammetry and remote sensing method (Chen et al., 2016). Full automation in the $3 \mathrm{D}$ data (i.e. $3 \mathrm{D}$ indoor and outdoor mapping) acquisition is the current trend and will be achieved in the near future. However, the current state-of-theart 3D mobile mapping equipment (Trimble, 2016) needs manual operation. Unmanned and fully automatic mobile mapping study is relatively rare.

Aim to solve the problem of automatic 3D data acquisition in indoor environment; this paper introduces a low cost multisensor robot laser scanning system that performs online 3D indoor mapping in real-time. The proposed robot system is designed to include 2D/3D imaging sensors (Laser scanner/ High resolution panoramic camera), position/orientation sensors (Odometer/MEMS-IMU (Micro-Electro-Mechanical SystemInertial Measurement Unit)) and two wheel differential chassis operating on ROS (Robot Operating System, www.ros.org/) to realize indoor mapping data acquisition without human intervention. The robot mobile mapping system can be applied to applications such as indoor scene visualization (Camplani et al., 2013), floor plan generation (Choi et al., 2015), BIM (Building Information Model) construction (Xiong et al., 2015), simulation (Gemignani et al., 2016), indoor navigation (Gutierrez-Gomez et al., 2016), virtual reality (Seibert et al., 2017) and etc.
The remainder of the paper is organized as follows: following the introduction, section 2 introduces the overall architecture of this robot system. Experimental studies on the accuracy investigation of $3 \mathrm{D}$ indoor mapping system are presented in Section 3, and conclusions are drawn in the final section.

\section{LOW COST MULTI-SENSOR ROBOT LASER SCANNING SYSTEM}

\subsection{System design and integration}

Our low cost multi-sensor robot laser scanning system is a measurement system consisting of a series of instruments that includes a laser scanner, an IMU, a panoramic camera, an onboard control unit and a customized two wheel differential chassis. Figure 1 is a snapshot of the system, and Table. 1 is sensor specifications.

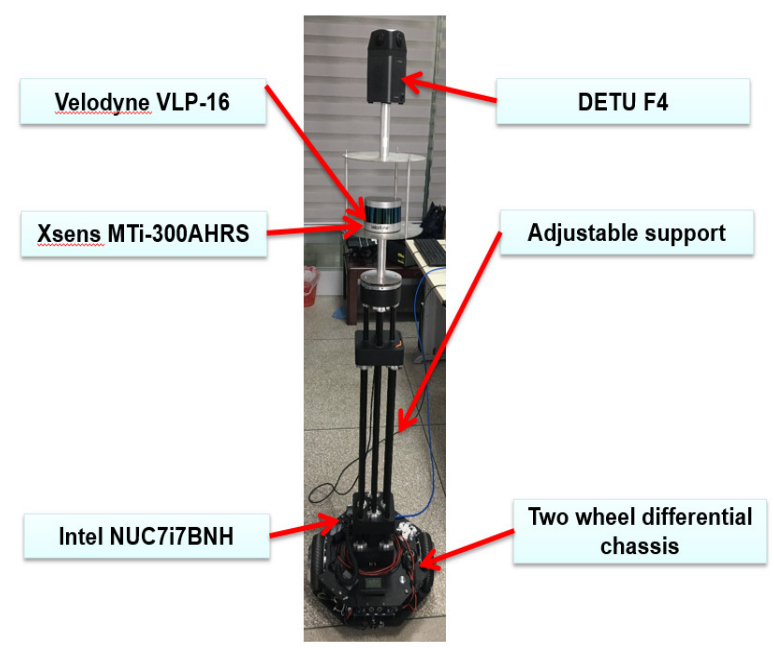

Figure 1. Snapshot of the low cost multi-sensor robot laser scanning system

\footnotetext{
* Corresponding author.
} 


\begin{tabular}{|c|c|}
\hline Sensor & Description \\
\hline Laser scanner & Velodyne VLP-16 \\
IMU & Xsens MTi-300AHRS \\
Panoramic camera & DETU F4 \\
On-board control unit & Intel NUC7i7BNH \\
\hline
\end{tabular}

Table 1. Sensor specifications

The laser scanner horizontally mounted in the system is a Velodyne VLP-16.The measurement rang of Velodyne VLP-16 is 100 meter, and the vertical FOV (Field of View) is $\pm 15^{\circ}$. The sampling frequency is $10 \mathrm{~Hz}$. The MEMS-IMU is an Xsens MTi-300AHRS that is tightly mounted rigidly with respect to the laser scanner. Its Gyro will produce a deviation of $10^{\circ}$ per hour, its output frequency is up to $2 \mathrm{kHz}$, and its latency is less than 2 millisecond. The panoramic camera is a DETU F4, which is mounted on the top of the system, and it can output $6500 \times$ 3250 pixel images with a pixel size of $1.55 \mu \mathrm{m}$ at $24 \mathrm{~Hz}$. The on-board control unit is an Intel NUC7i7BNH with an Intel Core i7 (4 M cache, up to $4.00 \mathrm{GHz}$ ) processor and $32 \mathrm{~GB}$ memory, which is the processing instrument of the system.

The synchronization of these instruments is done electronically. All data are produced with timestamp. Before processing, data from different sensors is combined using the timestamp information. The geometry calibration of the sensors are done mechanically, currently.

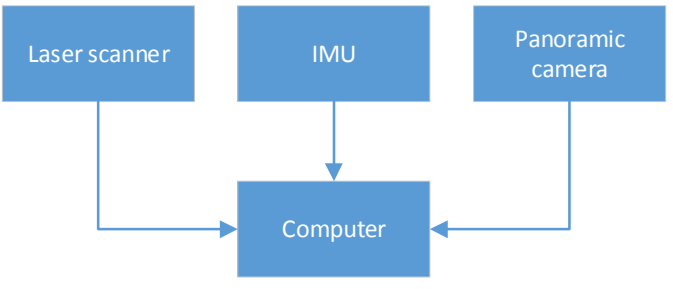

Figure 2. Instruments connections

\subsection{D point cloud generation by integration online 2D SLAM and IMU data}

2D SLAM (Simultaneous Localization and Mapping) GMapping (Giorgio Grisetti, et.al. 2007) is a highly efficient Rao-Blackwellized particle filer to learn grid maps from laser range data. A 2D occupancy grid map with laser and pose data collected by a robot can be solved by GMapping which is a $2 \mathrm{D}$ slam algorithm and only measures 3 DOF (Degree of Freedom) position and orientation parameters. In order to achieve full 6 DOF position and orientation parameters estimation, IMU data is used to measure the roll and pitch angle while the $\mathrm{z}$ parameter in the global mapping frame is solved in the light of the orientation and the height of the laser scanner with respect to the robot chassis. We can obtain 3 DOF position and orientation parameters that are $2 \mathrm{D}$ position and azimuth $(x, y, a)$ of the laser scanner by GMapping and roll/pitch $(r, p)$ by IMU. The height $(h)$ of the laser scanner can be easily measured. The only unknown POS parameter $z$ can be calculated as:

$$
z=\left\|h \cdot \boldsymbol{\eta}_{h} \cdot \boldsymbol{\eta}_{z}\right\|
$$

Where $\boldsymbol{\eta}_{\boldsymbol{h}}$ is the unit direction vector of the robot chassis in vertical direction, $\boldsymbol{\eta}_{z}=(0,0,1)^{T} . \boldsymbol{\eta}_{\boldsymbol{h}}$ is calculated corroding to the measured orientation parameters $(r, p, a)$. Once the 6 DOF parameters are determined, the laser point clouds are generated combining the range measurements derived from the laser scanner and the calculated position and orientation parameters.

\section{EXPERIMENTS AND ANALYSIS}

Experiments were undertaken to check the validity and effectiveness of the proposed low cost multi-sensor robot laser scanning system in real indoor scene including multiple rooms and a long corridor. The reference data for robot laser scanning data validation was collected by TLS (Terrain laser scanner) scanner Z-400. The VZ-400 is moved to multiple scan locations to gather scans and those scans are registered manually afterwards to construct the whole measured space in the form of very dense $3 \mathrm{D}$ point cloud. The robot navigates itself according to a predefined path to collect the 3D point clouds dynamically.

The point clouds collected by VZ-400 and the low cost multisensor robot laser scanning system are shown in figure 3 . It can be seen that point cloud collected by VZ-400 is much better than the point cloud collected by the system in terms of point density and completeness. The point cloud collected by the system is unevenly distributed, and its coverage is narrow, but the geometry of the indoor scene is well preserved.

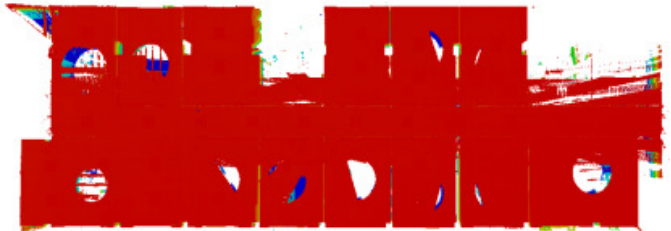

(a) Point clouds collected by Vz400

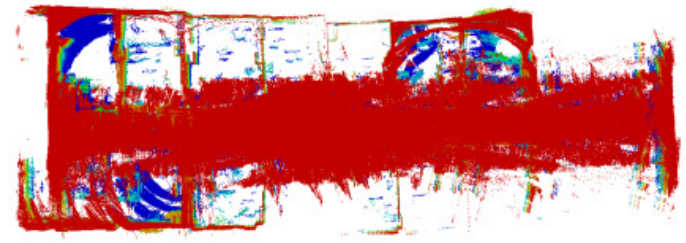

(b) Point clouds collected by robot system.

Figure 3. Point clouds of the experiment indoor scene

To analyse the accuracy of the point cloud collected by the system, the point cloud collected by the system is compare against to the reference data gathered by VZ-400 in terms of point-to-plane distance after these two point clouds are manually registered. The point-to-plane distance formula is given as follow:

$$
d=\left\|\eta_{i} \cdot\left(P_{\text {com }}^{i}-P^{i}\right)\right\|^{2}
$$

Where $\eta_{i}$ is the normal vector at $P_{r e f}^{i}, P_{c o m}^{i}$ is the compared point from the point cloud collected by the robot system, and $P^{i}$ is the reference point from the point cloud collected by VZ-400.

The comparison results are given by figure. 4 and table 2 . Figure. 4 depicts the calculated difference between the two point clouds in rendered color. Small differences are assigned with blue while large differences are colored red in the figure. As it can be learn from the rendered results, most of the points collected by the robot is consistent with the reference data qualitatively. Table 2 lists the quantitative statistic of the calculated differences that shows that the error of $99.2288 \%$ points in the robot point clouds is within 0.225 . 


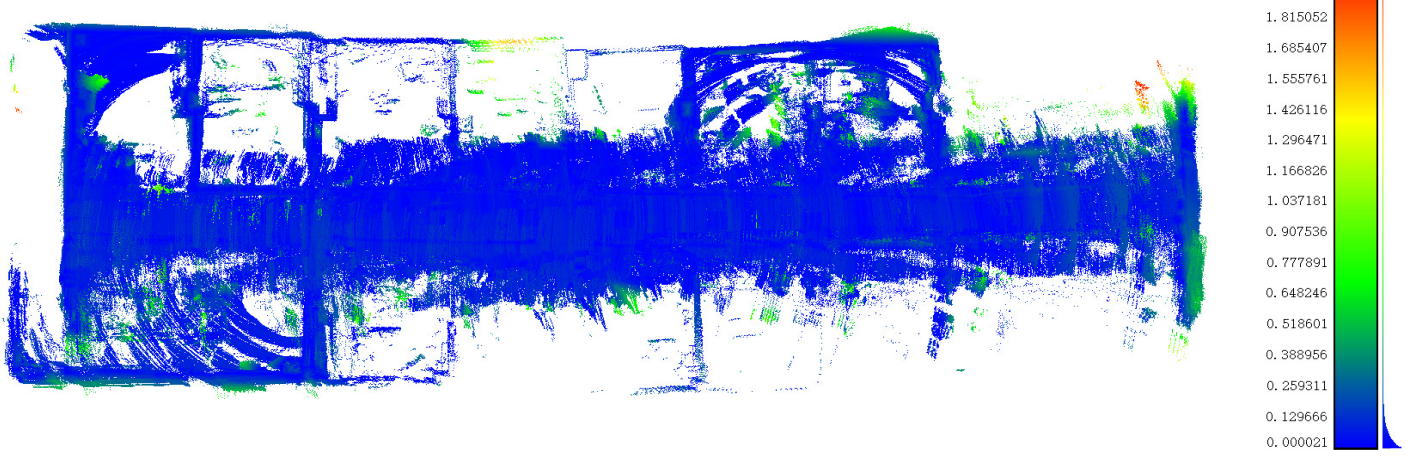

Figure 4. Comparison result of robot \&VZ400 point clouds in terms of point-to-plane distance rendered in color.

\begin{tabular}{|c|c|c|c|c|c|c|c|c|}
\hline Distance(meter) & $0 \sim$ & $0.225 \sim$ & $0.450 \sim$ & $0.675 \sim$ & $0.900 \sim$ & $1.125 \sim$ & $1.350 \sim$ & $1.575 \sim$ \\
& 0.225 & 0.450 & 0.675 & 0.900 & 1.125 & 1.350 & 1.575 & 1.800 \\
\hline Percentage (\%) & 99.228 & 0.7539 & 0.0109 & 0.0483 & 0.00077 & 0.00029 & 0.00016 & 0.00035 \\
& 8 & & & & & & & \\
\hline
\end{tabular}

Table 2. Distribution of point-to-plane distance of the compared point clouds

\section{CONCLUSION}

A low cost multi-sensor robot laser scanning system for 3D indoor data acquisition is proposed in this study, and the accuracy of its laser scanning point clouds are investigated. The experiment validates the effeteness of the proposed system for 3D indoor laser scanning applications. The proposed robot mobile mapping system is equipped with multiple sensors including a panorama camera, a laser scanner, and an IMU that are calibrated and synchronized to collect variety of data sources simultaneously. The $6 \mathrm{DoF}$ position and orientation parameters are estimated by merging the IMU and a $2 \mathrm{D}$ slam algorithm (GMapping). In conjunct with the range measurement from the laser scanner, the 3D point clouds are generated. The point clouds of a typical indoor scene generated by the proposed system are compared with ground truth data collected by a TLS scanner showing an accuracy of $99.2 \%$ below 0.25 meter. Although the density of the point clouds is relatively low when compared with the traditional TLS point clouds, in terms of the investigation results, the proposed robot system is applicative in applications such as automated indoor navigation/mapping and etc. In the future, the focus of following study will be putted on the improvement of the stability and accuracy of the 6 DoF position and orientation parameters estimation by combining both online SLAM and offline scan matching algorithm.

\section{ACKNOWLEDGEMENTS}

Work described in this paper is jointly supported by the National Science Foundation of China project under Grant No. 41701530 and No.41371431.

\section{REFERENCES}

Camplani, M., Mantecon, T. and Salgado, L., 2013. DepthColor Fusion Strategy for 3-D Scene Modeling With Kinect. IEEE Transactions on Cybernetics, 43(6), pp. 1560-1571.
Chen, C., Yang, B. S. and Song, S., 2016. Low Cost and Efficient 3d Indoor Mapping Using Multiple Consumer RGB-D Cameras. The International Archives of the Photogrammetry, Remote Sensing \& Spatial Information Sciences, 41.

Choi, S., Zhou, Q.-Y. and Koltun, V., 2015. Robust Reconstruction of Indoor Scenes. Proceedings of the IEEE Conference on Computer Vision and Pattern Recognition, pp. 5556-5565.

Gemignani, G., Capobianco, R., Bastianelli, E., Bloisi, D. D., Iocchi, L. and Nardi, D. 2016. Living with robots: Interactive environmental knowledge acquisition. Robotics and Autonomous Systems, 78, pp. 1-16.

Grisetti, G., Stachniss, C. and Burgard, W., 2007. Improved techniques for grid mapping with rao-blackwellized particle filters. IEEE transactions on Robotics, 23(1), pp. 34-46.

Gutierrez-Gomez, D., Mayol-Cuevas, W. and Guerrero, J.J., 2016. Dense RGB-D visual odometry using inverse depth. Robotics and Autonomous Systems, 75, Part B, pp. 571-583.

Saripalli, S., Montgomery, J. F., and Sukhatme, G., 2015. Visually guided landing of an unmanned aerial vehicle. IEEE Transactions on Robotics \& Automation, 19(3), pp. 371-380.

Seibert, J. and Shafer, D. M., 2017. Control mapping in virtual reality: effects on spatial presence and controller naturalness. Virtual Reality, pp. 1-10.

Thornton, S. M., Pan, S., Erlien, S. M. and Gerdes, J. C., 2017. Incorporating ethical considerations into automated vehicle control. IEEE Transactions on Intelligent Transportation Systems, 18(6), pp. 1429-1439.

Xiong, B., Jancosek, M., Elberink, S. O. and Vosselman, G., 2015. Flexible building primitives for $3 \mathrm{~d}$ building modeling. ISPRS Journal of Photogrammetry \& Remote Sensing, 101, pp. 275-290. 